\title{
RESIDUOS ELÉCTRICOS-ELECTRÓNICOS EN EL MODELO DE ECONOMÍA CIRCULAR: OPORTUNIDADES Y DESAFÍOS EN EL SECTOR
}

Por: Kevin David López Deodanes, Estudiante de tercer año de Ingeniería Industrial, UCA, 00014819@uca.edu.sv

Alex Alberto Saldaña Amaya, Estudiante de tercer año de Ingeniería Industrial, UCA, 00079819@uca.edu.sv

Pamela Saraí Palma Méndez, Estudiante de tercer año de Ingeniería Industrial, UCA, 00007019@uca.edu.sv

Valeria María Medina Sagastume, Estudiante de tercer año de Ingeniería Industrial, UCA, 00051719@uca.edu.sv

Diego Rafael Olmedo Pacheco, Estudiante de tercer año de Ingeniería Industrial, UCA, 00022219@uca.edu.sv

Verena Paola Zepeda Mercado, Estudiante de tercer año de Ingeniería Industrial, UCA, 00070519@uca.edu.sv

Ing. René Ernesto García Rivas, Docente Investigador del Departamento de Operaciones y Sistemas, UCA, regarcia@uca.edu.sv

\section{Resumen}

La presente investigación presenta un análisis de oportunidades y desafíos de aplicación y sustitución del modelo económico lineal por uno de visión circular comprometido con el medio ambiente, en el que se tome conciencia de los impactos que provoca la generación y el aumento de basura electrónica producida y consumida en todo el globo. Además, se discuten los beneficios y desafíos que conlleva el aplicar esta estrategia para transitar de un modelo de explotación de los recursos naturales hacia uno de explotación de los residuos y desechos para la producción y remanufactura de nuevos aparatos eléctricos y electrónicos.

\section{Palabras Clave}

Basura electrónica, economía circular, reciclar, remanufacturar

\section{Introducción}

Los dispositivos electrónicos son una herramienta útil tanto para el hogar como para las empresas, aun así, muchos son desechados en un contenedor de basura, otras se almacenan sin hacer ningún uso de ellas o simplemente se tiran a las calles, pensando que no poseen ningún valor o utilidad, cuando, de hecho, tanto dispositivos eléctricos como electrónicos están compuestos por materiales valiosos que pueden ser extraídos y remanufacturados con el propósito de darles un nuevo valor y uso.

El crecimiento exponencial del consumo y uso de aparatos tecnológicos no solo representa oportunidades para los seres humanos, sino también una diversa gama de amenazas, pues muchos de los productos que se han fabricado y se fabricarán, con el paso del tiempo, terminarán como basura que podría afectar gravemente el planeta. Es por eso que, mediante esta investigación, se pretenden identificar las oportunidades de transitar de una visión lineal a una circular en la producción y consumo de estos aparatos; un sistema que mejoraría diversos ámbitos de producción de nuevos productos. Otro de los propósitos de esta investigación es analizar y mostrar a diferentes audiencias - sociedad civil y gobierno- los beneficios de desarrollar el modelo de economía circular, entre los cuales se encuentran: la preservación del medio ambiente, el desarrollo económico, y la creación de empleos.

\section{Objetivos de la investigación}

\section{A. Objetivo general:}

1. Identificar oportunidades y desafíos en la aplicación del modelo de economía circular vinculada al fenómeno de generación de desechos electrónicos

B. Objetivos específicos:

1. Conocer las oportunidades de mercado y beneficios socioambientales a través del reciclaje de basura electrónica

2. Analizar el marco legal en materia de legislación salvadoreña de políticas eco amigables.

3. Indagar en el comportamiento de los salvadoreños frente al tratamiento de los residuos eléctricos y electrónicos 


\section{Marco referencial}

\section{A. Conceptos y definiciones}

El Foro Económico Mundial (2019) expresa que:

Los desechos electrónicos se definen como cualquier objeto con un enchufe, cable eléctrico o batería, que ha llegado al final de su vida útil, así como los componentes de estos. Los residuos electrónicos también se denominan residuos de aparatos eléctricos o electrónicos, o WEEE en inglés. (...) Los desechos electrónicos representan solo el $2 \%$ de los flujos de residuos sólidos, pero puede representar el $70 \%$ de los residuos peligrosos que terminan en el vertedero. De hecho, hasta 60 de los elementos de la tabla periódica se pueden encontrar en complejos equipos de la electrónica. (p.7)

\section{B. La basura electrónica en cifras}

Forti et al. (2020) revelan que, en 2019, el mundo generó 53,6 Mt de desechos electrónicos, un promedio de 7,3 kg per cápita. La generación global de desechos electrónicos creció 9,2 Mt desde 2014 y se prevé que crezca hasta 74,7 Mt para 2030, alcanzando el doble en solo 16 años. Las estadísticas muestran que en 2019 Europa fue el continente con la tasa más alta de recolección y reciclaje (42,5\%), seguido por Asia $(11,7 \%)$, América y Oceanía reportaron tasas similares (9.4\% y $8.8 \%$, respectivamente) y África tuvo la tasa más baja con un $0,9 \%$. Hay un $82.6 \%$ de residuos electrónicos en el año 2019 cuyo destino no es conocido al igual que su impacto ambiental.

\section{América y la basura electrónica}

Baldé et al. (2017) presentan que uno de los principales productores de desechos electrónicos del continente americano es Estados Unidos con 6,3 Mt. Seguido por Brasil, con 1,5 Mt, y México, con 1 Mt. Además, se estima que América Latina, en 2016, generó 4,2 Mt de desechos electrónicos, con una media de $7,1 \mathrm{~kg} / \mathrm{inh}$. Los países latinoamericanos con mayor generación de desechos electrónicos son: Brasil 1,5 Mt, México $1 \mathrm{Mt}$, y Argentina 0.4 Mt. Los tres primeros países de América Latina con la mayor generación de desechos electrónicos en cantidades relativas en 2016 fueron Uruguay (10,8kg /hab), Chile (8,7 kg /hab) Argentina $(8,4 \mathrm{~kg} / \mathrm{hab})$.

\section{Inserción del modelo circular para residuos electrónicos}

Una economía circular es un sistema en el que todos los materiales y los componentes se mantienen en su valor más alto en todo momento y los residuos se diseñan fuera del sistema. Puede ser considerada como opuesta a la economía lineal actual (Foro Económico Mundial, 2019).

Una economía circular par los materiales y componentes electrónicos y eléctricos maximizaría la cantidad de desechos electrónicos valiosos para la producción de nuevos productos y componentes electrónicos, lo que permitiría una mayor productividad de nuestros escasos recursos naturales. Esto contribuiría a modernizar la economía y a fortalecer una civilización ecológica (Lauren \& Pennington, 2018).

Un caso de una empresa con un modelo de economía circular es Apple, una de las empresas con más producción electrónica. En su portal de noticias, Apple Newsroom (2020), afirman que desmontan alrededor de 1.2 millones de dispositivos que los usuarios entregan cada año y los renuevan gracias a los programas de reciclaje que esta empresa ha realizado en los últimos años. Con esta iniciativa Apple logró evitar que más de 48,000 toneladas métricas de residuos electrónicos terminaran en los vertederos, además, desarrolló programas de laboratorios de recuperación de materiales con los cuales buscan optimizar el reciclaje de desechos electrónicos y reutilizarlos para reducir el impacto ambiental que esta gran empresa genera cada año.

Por otro lado, Lauren y Pennington (2018) manifiestan que China es otro país que ya ha definido objetivos sobre una economía circular. Plantean que el Consejo de Estado ha establecido un programa de implementación de un sistema dirigido a fomentar la responsabilidad del productor que establece objetivos que buscan que el $20 \%$ de contenido reciclado se usen para elaborar productos electrónicos y reciclar el $50 \%$ de todos los desechos electrónicos para 2025 , consolidando así su ruta hacia una economía circular en la industria electrónica.

\section{E. Cinco acciones de la industria para acelerar la transición hacia una economía circular}

Una de las principales preguntas alrededor de un modelo de economía circular es cómo comenzar a avanzar de un modelo lineal hacia allá. La Ellen MacArthur Foundation (2018) propone una serie de principios de la economía circular orientados a cambiar la forma en que diseñamos, elaboramos y usamos los productos electrónicos.

1. Diseño para circularidad: seleccione una estrategia de diseño circular que se ajusta al 
modelo de negocio, centrándose en preservar el valor

2. Dar un propósito adicional a la migración a la nube

3. Los productos se adaptan a las necesidades cambiantes

4. Incrementar la eficiencia del mercado de la reutilización

5. Aumentar la automatización del desmontaje y la remodelación de procesos

\section{F. Oportunidades y desafíos en el sector}

La aplicación de un modelo de economía circular en la industria electrónica puede traer diversos beneficios y desafíos. Uno de los principales son los beneficios económicos y la creación de empleo. El Foro Económico Mundial (2019) afirma que "hay 100 veces más oro en una tonelada de teléfonos inteligentes que en una tonelada de mineral de oro. (...) Los depósitos más ricos de materiales valiosos de la tierra se encuentran en vertederos o casas de personas". Por su parte, Lauren y Pennington (2018) proponen que una economía circular en la industria electrónica podría reducir los costos para los consumidores en un $7 \%$ para el 2030 y un $14 \%$ para el 2040 . Avanzar hacia una economía circular en la industria también tiene el potencial de creación de empleos. El Foro Económico Mundial publicó que emprendedores y cooperativas de diversos países están experimentando reciclar los derechos electrónicos de sus operaciones, procesos que ya están generando puestos de trabajo dignos, con condiciones de formalidad en la cadena de valor de los residuos electrónicos. Agregan que "con la combinación de políticas adecuada y acceso a finanzas, dichos enfoques podrían ampliarse, generando trabajos adicionales para decenas, sino cientos de miles de trabajadores de la economía circular. (Foro Económico Mundial, 2019).

Otro de los beneficios de este tipo de economía son los medioambientales. El reciclaje de hierro, aluminio, y el cobre contribuyen a un ahorro neto de $15 \mathrm{Mt}$ de $\mathrm{CO} 2$, equivalente a las emisiones del reciclaje de materias primas secundarias sustituidas por materias vírgenes. Al reciclar los desechos electrónicos los países podrían, al menos, mitigar su demanda material de una manera segura y sostenible (Forti et al., 2020).

El modelo de economía circular también plantea retos, sobre todo en términos de producción y legislación. En cuanto a producción uno de los principales desafíos es que de los materiales reciclados se pueda hacer, efectivamente, un producto completamente nuevo, pues pueden llegar a estar compuestos por más de mil sustancias diferentes. (Foro Económico Mundial, 2019).

Al mismo tiempo Forti et al. (2020) hacen constar que:

Desde la perspectiva del diseño de materiales AEE el proceso es muy complejo, puesto que, hasta 69 elementos de la tabla periódica se puede encontrar en $\mathrm{AEE}$, incluidos los metales preciosos (por ejemplo, oro, plata, cobre, platino, paladio, rutenio, rodio, iridio y osmio); materias primas críticas como cobalto, paladio, indio, germanio, bismuto y antimonio; y metales no críticos, como aluminio y hierro. (...) Por otro lado, el sector del reciclaje a menudo se enfrenta a altos costos de reciclaje y desafíos en el reciclaje de materiales. (Forti et al., 2020, p. 58)

En cuanto a legislación, Baldé et al. (2017) presentan algunos retos en materia de legislación. Entre los principales retos están que los desechos electrónicos deben llegar a contribuir a la elaboración de un mejor producto, que sean más fáciles de reparar o, mejor aún, que sean más duraderos. Otro de los retos es que las políticas deben reforzar el uso eficiente de recursos y recuperación de materiales valiosos incorporados en los AEE; en América Latina el reto adicional es que los procesos legislativos aumenten su velocidad. $Y$ para los países con políticas y marcos normativos ya existentes el reto es acelerar su cumplimiento e implementación.

\section{G. Contexto reciente en El Salvador}

Desde hace algunos años varias empresas en el país están organizando campañas y proyectos para recolectar residuos electrónicos y darles un tratamiento adecuado. Por ejemplo, hace cuatro años la empresa de Movistar y la distribuidora de electricidad DELSUR en El Salvador se unieron en un programa de reciclaje de residuos electrónicos llamado "La tierra te llama, contesta" que consistió en incentivar la cultura del reciclaje por medio de la recolección de baterías de celulares, celulares y cargadores en desuso (Fundemas, 2020). Por otro lado, de acuerdo con la Prensa Gráfica (2016), algunas empresas salvadoreñas como Siman se han sumado a campañas para recolectar este tipo de residuos. Implementada desde 2011 esta campaña consiste en que la ciudadanía lleve los aparatos en desuso a puntos de recolección para así evitar que se desechen de manera inadecuada; para el año 2016 se había logrado recaudar más de 35 toneladas de desechos. 
El Salvador, por otro lado, no posee normativa específica para la regulación de la importación de aparatos electrónicos ni tampoco para el manejo de los residuos de este tipo que se generen tanto en hogares, empresas e instituciones. El país se sujeta a lo establecido en los tratados internacionales suscritos y a lo establecido en el Reglamento Especial para el Manejo de Desechos y Residuos Peligrosos (Meléndez, 2018).

El acercamiento hacia esta economía más reciente por parte de legislaciones se hizo en el 2019 por medio de la propuesta de ley llamada "Ley de Gestión Integral de Residuos y Fomento al Reciclaje", busca crear ciudades sustentables, en armonía con el medio ambiente. Además, a través de la misma se busca fomentar la formalización y capacitación de microempresas, cooperativas y otras organizaciones que trabajan en la recuperación, separación, tratamiento, reciclaje o gestión integral de residuos; así como la inclusión de nuevos actores para prestar servicios al sistema (Serrano, 2019).

\section{Hipótesis}

Este trabajo se ha realizado a partir de una hipótesis compuesta por tres ideas fundamentales:

-La población adulta joven tiende a depender de los productos eléctricos-electrónicos, pero tiene poca conciencia del manejo de estos tras haber rendido su vida útil

- La población salvadoreña tiende a desconocer el marco legal en políticas de reciclaje y tratamiento de desechos sólidos

-La población salvadoreña desconoce las campañas de recolección de residuos electrónicos en el país

\section{Metodología de la investigación}

Para el proceso de esta investigación se optó por el tipo de investigación no experimental, con un enfoque cuali-cuantitativo y estudio de carácter descriptivo. Se pretende contemplar las oportunidades y desafíos en la inserción del modelo de economía circular como estrategia para la reutilización de la basura electrónica, de forma que se aproveche el potencial que tienen los residuos eléctricos-electrónicos, no solo como forma de innovación en material de negocios, sino también, económica, social, jurídico y ambiental, áreas dentro de la realidad que necesitan cambios radicales, mayor asequibilidad a productos tecnológicos, innovadores, económicos y de calidad, reducción de brechas tecnológicas, promulgación de leyes en torno al rápido crecimiento de tecnologías cada vez más utilizadas, y políticas eco-amigables como respuesta para combatir las crisis medioambientales.

\section{Instrumentos de investigación}

En el desarrollo del estudio se hará uso de encuestas como instrumento de recolección de datos para tener una percepción más cercana de la realidad salvadoreña en torno al comportamiento de la ciudadanía frente a las prácticas de conciencia medioambiental. Adicionalmente, se realizará una entrevista a un profesional en el área de economía circularJosé Ricardo Flamenco, mediante la cual se pretende un análisis profundizar en para cumplir los objetivos antes propuestos, a modo de contrarrestar la teoría y los resultados obtenidos en los formularios con una opinión profesional.

\section{Población y muestra}

Los sujetos por investigar son población adulta joven de entre 20 a 30 años de El Salvador, elegidos al azar, sin considerar su sexo o las diferencias entre edades o entre perfiles económicos, sociales, políticos o medioambientales. Se realizaron 71 encuestas y una entrevista a profundidad.

\section{Análisis e Interpretación de datos}

\section{A. Resultados de encuestas}

A continuación, se presentan los resultados obtenidos en las 71 encuestas realizadas a la población adulta joven salvadoreña.

\section{Aparatos electrónicos usados con mayor frecuencia}

La mayor parte de los encuestados hacen uso con mayor frecuencia de su celular, en segundo lugar, se encuentra la computadora, en tercer lugar, la televisión y en último lugar la tableta electrónica. Se concluye que las personas tenderían a desechar y/o reemplazar con más frecuencia teléfonos móviles ya que es el más utilizado. La computadora se vuelve el segundo que se desecharía con más frecuencia. La figura 1 muestra estos resultados. 


\section{Gráfico 1.}

Frecuencia de uso de AEE

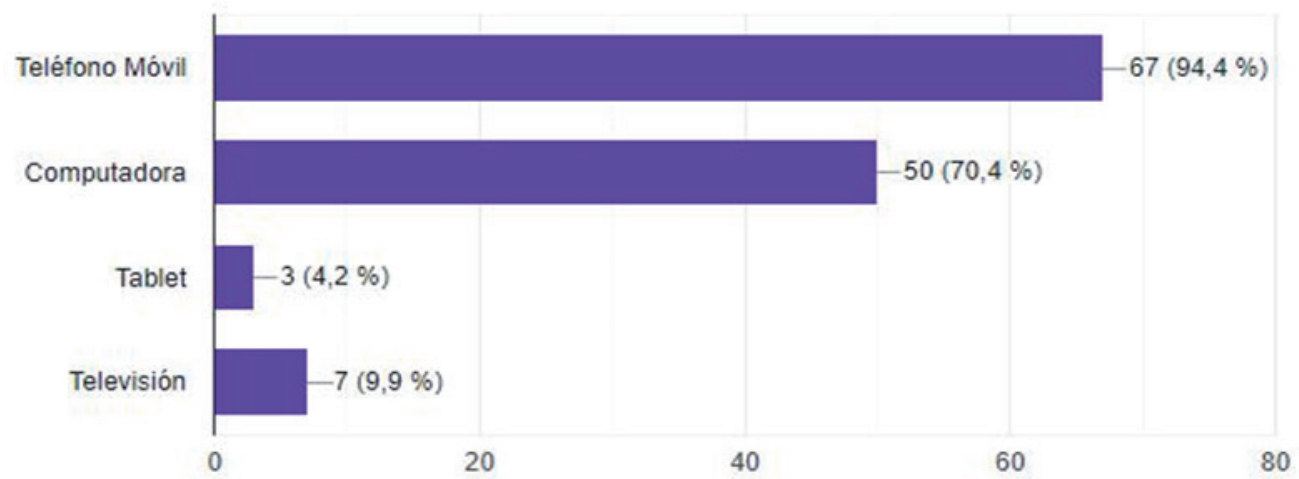

Fuente: Elaboración propia

\section{Razones para reemplazar sus aparatos electrónicos}

El $70 \%$ de la población encuestada decide reemplazar sus AEE debido al deterioro (obsolescencia del programa), mientras que $18 \%$ lo hace por un nuevo y mejor sistema operativo que satisfaga sus necesidades. Mientras que un $7 \%$ de la población encuestada dice que el reemplazo de su equipo electrónico se debe a la pérdida o robo del mismo. En menor parte, las razones son debido a que se desea

\section{Gráfico 2.}

Razones para reemplazar AEE

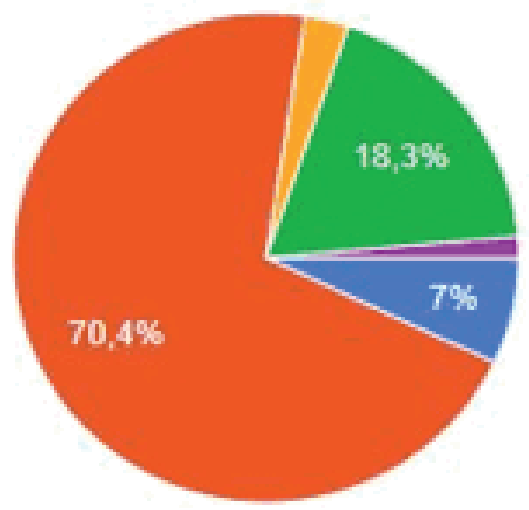

Fuente: Elaboración propia

\section{Prácticas para deshacerse de los aparatos electrónicos y eléctricos}

A partir de la figura 3 se observa que las tácticas puestas en marcha por la población encuestada para deshacerse de este tipo de aparatos que ya no le son de utilidad están.

1) Con un $60.6 \%$, la estrategia más utilizada por la población consiste en guardar los aparatos electrónicos que ya no utiliza estar al día con las nuevas tendencias o debido a que se arruine por un mal uso. Por tanto, se concluye que, el crecimiento acelerado de las herramientas tecnológicas y la dependencia de estas, hacen que los aparatos electrónicos sean fundamentales en el diario vivir de la mayoría de la población, lo cual se evidencia no solo en quienes reemplazan sus equipos por necesidad de un mejor sistema operativo, sino también por quienes lo hacen debido a la obsolescencia. La figura 2 muestra dichos resultados.

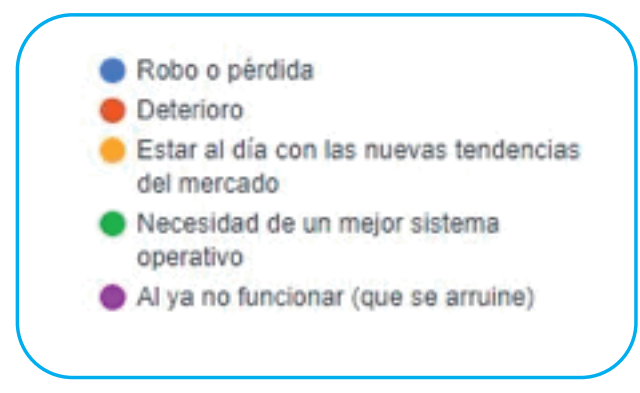

2) Un $42.3 \%$, vende los aparatos electrónicos que ya no le son de utilidad

3) Un $28.2 \%$, donan o regalan a otra persona estos aparatos para que le sea de utilidad.

4) Mientras que las prácticas que son menos frecuentes consisten en: entregar a una persona o empresa recicladora de este tipo de desechos (11.3\%); botar los aparatos electrónicos junto a basura tradicional (5.6\%); orienta sus decisiones en función del estado del aparato (1.4\%). 


\section{Gráfico 3.}

Prácticas utilizadas por la población para deshacerse de los AEE

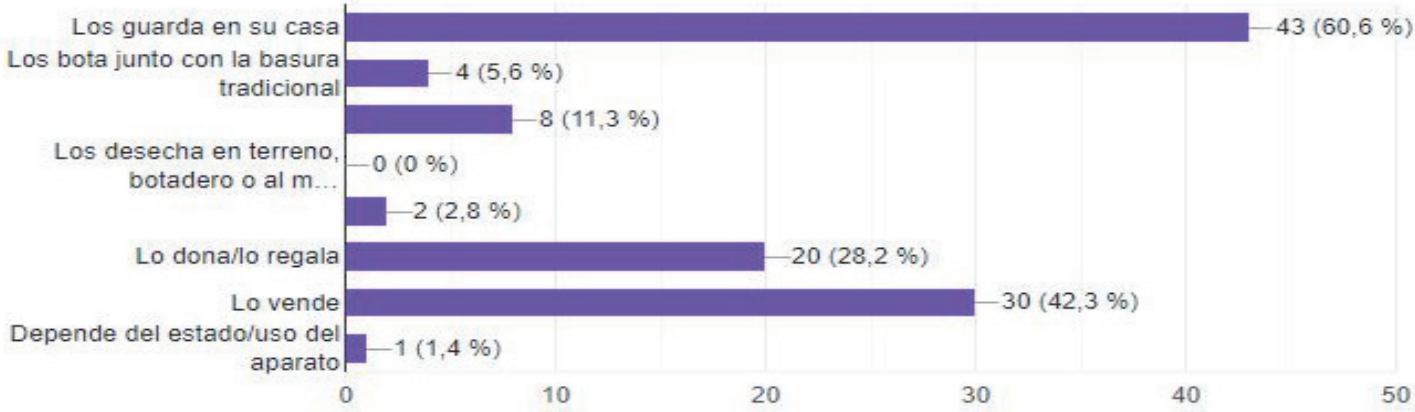

Fuente: Elaboración propia

Por lo que, a partir de la frecuencia de las prácticas para el tratamiento de estos dispositivos se puede concluir que la mayoría de la población desconoce las formas para deshacerse de estos y decide guardarlos en sus casas. Por otro lado, un buen porcentaje de la población, decide poner en práctica estrategias de reutilización de los aparatos, ya que, decide venderlos o regalarlos; mientras que, en menores cifras, la población los entrega a empresas recicladoras, y otras deciden botarlos junto a basura tradicional.
Cabe resaltar que, en esta pregunta, las personas encuestadas eran libres de marcar más de una opción.

Información sobre campañas de recolección de desechos electrónicos en El Salvador

La figura 4 muestra que el $93 \%$ de los encuestados no han participado o escuchado de alguna campaña de recolección de desechos electrónicos en el país, frente a un $7 \%$ de la población que sí tiene conocimiento de estas campañas.

\section{Gráfico 4.}

Participación ciudadana en campañas de recolección de AEE

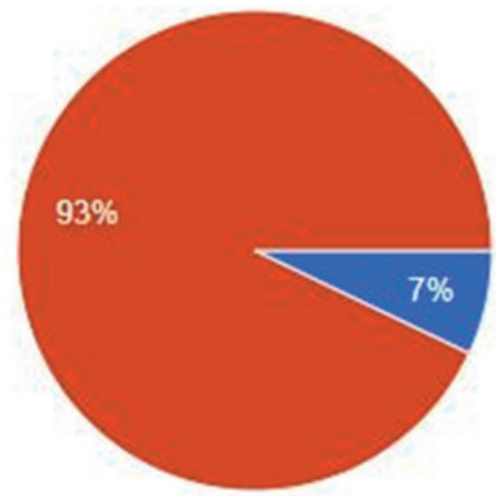

Fuente: Elaboración propia
El gráfico muestra que, el 93\% de los encuestados no han participado o escuchado de alguna campaña de recolección de desechos electrónicos en el país, frente a un $7 \%$ de la población que sí tiene conocimiento de estas campañas.

Se concluye así que la gran mayoría de la población desconoce de prácticas dedicadas a la reducción de este tipo de desechos llevadas a cabo en el país.
Conocimiento de leyes o normativas en referencia a la reducción o manejo de residuos electrónicos

La figura 5 revela que el $94.4 \%$ de las personas encuestadas no conocen ninguna ley o normativa nacional que haga referencia a la reducción y/o manejo de residuos electrónicos, frente a $5.6 \%$ que sí conocen el marco legal para contrarrestar el impacto generado por el aumento de desechos electrónico. 


\section{Gráfico 5.}

Conocimiento de leyes o normativas nacionales sobre el manejo de AEE.

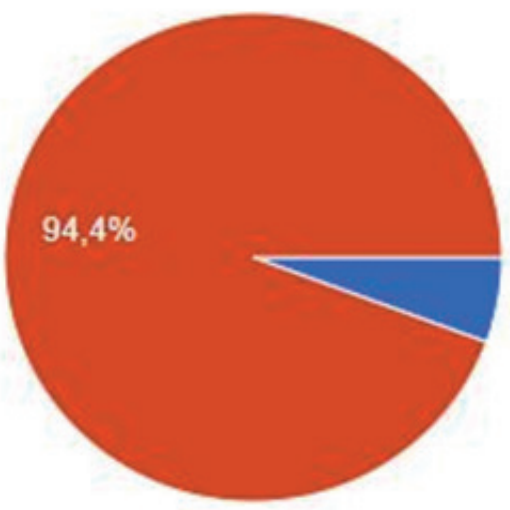

Fuente: Elaboración propia

Si bien nuestro país no cuenta con ningún tipo de regulación o ley para frenar el crecimiento de este tipo de desechos o tratar residuos electrónicos, sí cuenta con algunas normativas y reglamentos para la regulación o tratamiento de desechos sólidos en general. Así como guías de acción empresarial, industrial y ciudadanas. Sin embargo, falta formular leyes o marcos regulatorios adecuados al tratamiento de este tipo de residuos electrónicos y eléctricos y asegurar un estricto cumplimiento de las mismas en favor del medio ambiente.

\section{Necesidad de formular leyes para la reducción y/o} manejo de los desechos electrónicos

En la figura 6 se puede observar que el $98.6 \%$ de la población encuestada considera que el país deba formular leyes para la reducción y/o manejo de los residuos electrónicos, mientras que un $1.4 \%$ está en oposición a ello.

\section{Gráfico 6.}

Necesidad de formular leyes para reducir y manejar los desechos electrónicos

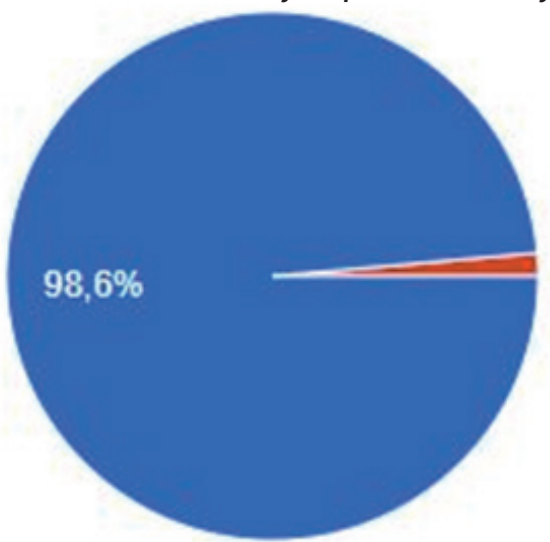

Fuente: Elaboración propia

A partir de los resultados, se puede concluir que, la de la población encuestada está a favor de políticas públicas en nuestro país para controlar y frenar lo mejor posible estos desechos.

\section{Factibilidad de reutilizar AEE para fabricación de nuevos productos}

La figura 7 muestra que la gran mayoría de la población encuestada (97.2\%) está de acuerdo con que los equipos electrónicos en desuso deben ser reutilizados con un fin ecológico y que esto es crucial para reducir el impacto medioambiental que tienen las empresas que producen estos equipos. 


\section{Gráfico 7.}

Necesidad de reutilizar los equipos electrónicos en desuso para la fabricación de nuevos productos

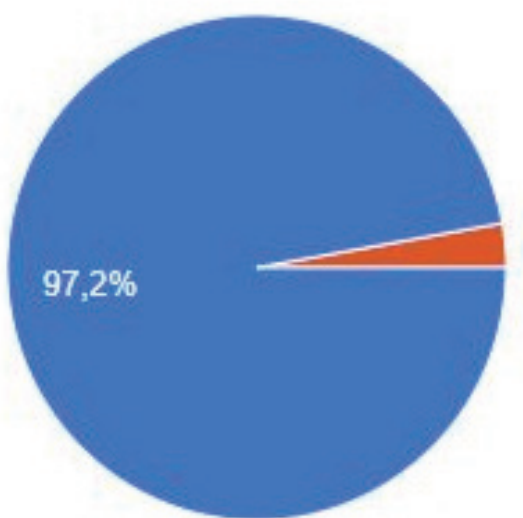

Fuente: Elaboración propia

\section{Disposición a consumir productos manufacturados a partir de desechos electrónicos}

Según los resultados el $62 \%$ de las personas encuestadas están dispuestas a adquirir los productos hechos con materiales reciclados de basura electrónica mientras que el resto se muestra con cautela sobre dichos productos. Una cantidad considerable de la población encuestada aún no tiene los insumos de confiabilidad para consumir productos reciclados por diferentes factores, como, por ejemplo, no tener mucha información sobre el tema ni conocer sobre los estándares de calidad que dichos productos representan o tienen que representar. Aunque la mayoría de las personas encuestadas están de acuerdo en reutilizar los equipos no todas están seguras de comprar el producto final a partir de estos.

\section{Gráfico 8.}

Disposición a consumir productos manufacturados a partir de desechos electrónicos

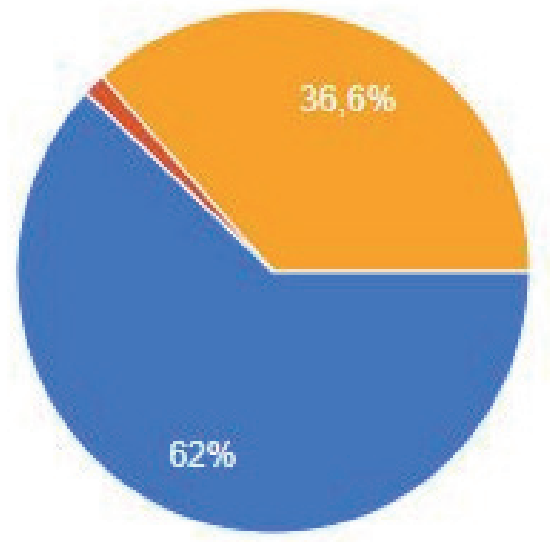

Fuente: Elaboración propia

\section{A. Entrevista: Descripción del fenómeno}

Se entrevistó a José Ricardo Flamenco, Licenciado en ciencias de la comunicación graduado de la universidad Dr. José Matías Delgado, trabajó en Termoencogibles, y estuvo involucrado en el proyecto de recicla 503, actualmente desempeñándose como consultor medioambiental y de economía circular. En la entrevista se abordaron dos ejes principales: medioambiental y económico; desde la perspectiva y experiencia profesional del Lic. Flamenco.

En el aspecto medioambiental, bajo la pregunta “¿Cómo percibe la cultura en el país con respecto al reciclaje?" el licenciado comenta que "Aquí en el país no hay una cultura de reciclaje, y ese es el mayor reto en cuanto a querer implementar una economía circular o querer aprovechar los recursos de la economía 
circular... si queremos empezar a implementar una cultura de reciclaje es algo que se debe empezar a hacer desde los niños más chiquitos, empezarles a enseñar a dónde tiene que ir cada cosa, los colores, cómo se tiene que ir depositando... el problema es que somos bien cómodos ... Siempre tenemos la cultura lineal: utilizo, desecho, utilizo, desecho; ... con los desechos electrónicos, es justamente lo que pasa... Estaba leyendo que los materiales electrónicos..., al destaparlos, todos los filamentos metálicos que tiene se pueden reutilizar para la creación de otros materiales... de modo que ya no es necesario que vayas a explotar recursos naturales para extraer materiales vírgenes y poder transformarlos... Con el reciclaje y la economía circular se pretende evitar que todos los materiales que tienen una segunda oportunidad de vida no se vayan para la basura normal".

Hace mención de los impactos que la falta de cultura del reciclaje significaba para el medio ambiente y los impactos que se tienen dentro de la calidad de vida de las personas. El licenciado también hacía la recomendación de, para poder inculcar una cultura de reciclaje de forma personal (Sin la necesidad de campañas de concientización) es el practicar el reciclaje de manera habitual, con los distintos grupos sociales, ya que, de esta manera, se podrá motivar desde los grupos pequeños, hasta llegar a los más grandes.

En el aspecto económico, bajo las preguntas “¿cómo cree usted que el implementar el sistema de economía circular ayudaría a la optimización de los recursos?, ¿qué recomendaría a la sociedad civil y a las empresas?" Para comenzar, el Lic. Flamenco hace hincapié en un cambio de mentalidad "Con esta situación de pandemia muchas empresas no creían en la cuestión del teletrabajo... la mentalidad está cerrada en eso, la pandemia obligó a muchas empresas a restructurar eso y empezar a innovarse en la cuestión del teletrabajo, es lo mismo con el reciclaje... porque el reciclaje y la economía circular comienza desde el momento en el que se tiene el cambio de pensamiento... Empezar a tener ese cambio de pensamiento va a traer grandes beneficios... A las empresas les sale caro andar comprando materia prima virgen para estar generando más recursos... En Termoencogibles, con este plástico que está recuperando se está ahorrando estar comprando más cantidad de material virgen, se está ahorrando en gastos... y los ahorros se invierten en mejorarías de la infraestructura... Todo esto, muchas empresas ya lo están aplicando, se están ahorrando para invertirlo en mejores cosas, porque si lo estuvieran haciendo en un ciclo normal de producción... en el tiempo, se tarda más tener el capital para poder invertirlo". Menciona, además, que la falta de tecnologías para procesar y re manufacturar productos hace pensar que muchos materiales no puedan ser reciclados, cuando en realidad sí es posible, como en el caso de las viñetas de botellas de agua.

"A los empresarios puede no interesarles ahorita, pero cuando se den cuenta que en el tiempo van a empezar a depender de recursos que ya estaban produciendo y que no pueden sacar más material de la tierra porque se lo acabaron, o en algún momento se acabó, ahí sí habría un problema, y van a empezar a querer aplicar el sistema circular, sobre todo en los modelos de producción... A la empresa misma le favorece (aplicar modelo circular) porque le generaría una mejor imagen ante la sociedad, ante sus compradores y proveedores... como una empresa socialmente sostenible... Con la gente... si empiezas a reciclar te quedas con el dinero, todos lo ven de forma monetaria, pierden de vista la perspectiva de salud y medio ambiente... Cambiar esa cultura es el gran reto". También un punto de sumo interés para nuestra investigación, pues menciona que en el país hay empresas que reciclan residuos electrónicos y los entregan a recicladores, pero desconocen qué es lo que hacen los recicladores con los desechos, lo que en realidad conlleva a un problema de suma importancia, pues es muy importante conocer el destino final que tendrán los residuos, ya que, se desconoce si serán tratados adecuadamente o si en cambio se aprovecharán de manera maliciosa de los residuos electrónicos. Es muy importante, por tanto, que las empresas sean socialmente responsables y que se cuenten además con los permisos legales y jurídicos para un trabajo honesto dentro del sector.

Para abonar a la temática, el licenciado comentaba, además, que para poder implementar el modelo de economía circular había que tener un cambio de mentalidad. Si bien es cierto que la economía circular supone buenas ventajas $i$ en realidad es factible para una empresa el implementar este tipo de sistema de producción? El licenciado refirió varios aspectos a considerar, por ejemplo, el costo de las maquinarias que procesarán el material a reciclar, verificar si en realidad es factible o no implementarlo, porque sucede que muchas veces no es factible y se incurre en gastos que innecesarios, todo depende de las posibilidades de la empresa y si estos cambios en realidad significarán una mejora sustancial para la empresa. 


\section{B. Análisis de hipótesis formuladas}

A continuación, se presenta cada una de las hipótesis formuladas y el análisis respectivo a partir de las evidencias mostradas en los apartados anteriores:

1. Dependencia de productos eléctricoselectrónicos, y poca conciencia del manejo de estos tras haber rendido su vida útil.

Tras los resultados obtenidos en las primeras tres preguntas de la encuesta, se concluye que hay una clara dependencia de los AEE y poca conciencia en el manejo de desechos, pues la mayoría opta por guardarlos en sus casas, salvo una parte de la población que tiende a reutilizarlos, por lo que, la hipótesis antes formulada se cumple.

2. Desconocimiento del marco legal en materia de tratamiento y reciclaje de desechos sólidos:

Tras los resultados obtenidos en algunas preguntas de la encuesta, se evidencia que la gran mayoría de la población no conoce políticas en materia de reciclaje y tratamiento de desechos sólidos y de AEE. Demuestran estar a favor de promulgación de leyes por parte del gobierno, por lo que, la hipótesis antes formulada se cumple.

3. Desconocimiento de campañas de recolección de residuos electrónicos en el país:

A partir de los resultados en la pregunta cuatro de la encuesta, se identifica que la gran mayoría de la población desconoce campañas de recolección de RAEE, por lo que, la hipótesis antes formulada se ha cumplido.

\section{Conclusiones}

1. La legislación salvadoreña frente a temas de reciclaje y economía circular es nueva e inexperta en comparación de otros países, como, por ejemplo, Costa Rica, Panamá y República Dominicana. Se debe trabajar en conjunto con organizaciones internacionales y en la formación de alianzas para llevar al país a la industria del futuro, en el que los sistemas o modelos lineales cambien de paradigmas, y se vuelvan amigables con el medio ambiente.
Además, El Salvador debe avanzar en materia de legislación, ya que no cuenta con muchas normativas sobre desechos sólidos y no necesariamente son de carácter obligatorio, debe trabajar para que las industrias y empresas respeten los recursos del país y que no pongan en riesgos a su ciudadanía. Se debe trabajar en diálogo junto a la asociación de empresas privadas para, no solo regular sus impactos, sino también para incentivar a sus usuarios y su personal para hacer más fácil y profunda el cambio de paradigma. Hay que aprovechar que la mayoría de la población se encuentra a favor de la promoción de políticas eco amigable $s$ y es consciente de la crisis medioambiental que sufrenuestro planeta y de los impactos de las tecnologías en nuestro medio. Así avanzar en materia de legislación y responsabilidad ciudadana debe ser una prioridad para el gobierno y las y los legisladores.

2. En general, la población desconoce del tratamiento que se le debe dar a este tipo de desechos, y, si bien se ponen en práctica estrategias eco amigables como la reutilización de estos aparatos a partir de ventas o donaciones, se desconoce $y$ se es incierto el final de estos dispositivos tras haber sido entregados a otro ser humano. La mayoría de las personas, al no saber cómo desechar los AEE deciden tenerlos guardados en sus casas, sin saber que pueden tener un nuevo valor a partir de un proceso de remanufacturación, pues contiene elementos valiosísimos como plata, oro, cobre, tierras raras, entre muchos otros. Esto significa una oportunidad, tanto para la ciudadanía misma, como para empresas que quieran emprender en este sector.

3. La implementación de un modelo de economía circular no solamente tiene repercusiones positivas desde el punto de vista económico de una empresa, sino que también un impacto en la reputación de la empresa, pues representaría una idea de negocio que es socialmente comprometida. Esto generaría doble beneficio, por un lado, 
buena publicidad y marcaría la diferencia entre la competencia y, por el otro lado, se darían nuevos ciclos de vida a la materia prima virgen transformándola en material reciclado que, a la larga, trae consigo beneficios hacia el medio ambiente disminuyendo la explotación de los recursos naturales.

4. A las empresas, este sistema de producción puede generarles múltiples beneficios, entre los cuales tenemos la optimización de los recursos, aplicando la ideología de 'zero waste' o 'cero desperdicios' durante todo el ciclo de vida de un producto; lanzamiento al mercado, compra, utilización por parte del cliente y finalmente el desecho de dichos productos cuando estos ya no son útiles o funcionales para el usuario o el consumidor. Lo que se busca es cerrar este ciclo, recolectando estos desperdicios, para hacerlos pasar por diferentes procesos de reciclaje, para que estén listos para ser convertidos en un nuevo producto o en el mismo.
5. El avance tecnológico y el constante proceso de evolución de los seres humanos ha permitido mejorar la vida de muchas personas alrededor del mundo, sin embargo, las prácticas irresponsables hacia el planeta y el ecosistema tienen sus consecuencias. Actualmente, nuestro planeta se ve afectado por la creciente generación de basura y con el aumento de un nuevo tipo o grupo de basura, los desechos eléctricos y electrónicos, lo que ha provocado una masiva producción de $\mathrm{AEE}, \mathrm{y}$ de e-waste. No obstante, y con el transcurso de los años, se determinó que una solución a este tipo de problemas es la implementación de un sistema de economía circular basado en la extracción de materias primas, procesamientos, entregas, uso, y reciclaje de sus desechos para la producción de nuevos productos con base eco amigables, lo que implica retos y oportunidades en los sectores sociales y empresariales: legislación, ambientales, sociales, económicos y de producción.

\section{Referencias}

Apple Newsroom. (2019, 8 de abril), Apple Expande Los Programas De Reciclaje Global. Apple Newsroom. https://www.apple.com/la/newsroom/2019/04/apple-expands-global-recycling-programs/

Meléndez, C.E. (2018). Resumen del diagnóstico de la situación actual de los residuos electrónicos en El Salvador. Proyecto Estrategia sostenible de gestión de residuos electrónicos en El Salvador y Panamá. Ministerio de Medio Ambiente y Recursos Naturales.

http://cidoc.marn.gob.sv/documentos/resumen-del-diagnostico-de-la-situacion-actual-de-los-residuoselectronicos-en-el-salvador-proyecto-estrategia-sostenible-de-gestion-de-residuos-electronicos-en-elsalvador-y-panama/

Ellen MacArthur Foundation. (2018). Circular Consumer Electronics, An Initial Exploration. Ellen MacArthur Foundation. https://www.ellenmacarthurfoundation.org/assets/downloads/Circular-ConsumerElectronics-2704.pdf

Fundemas (2020). Telefónica lanza iniciativa de reciclaje: “ila tierra te llama, contesta!”. https://www. fundemas.org/noticias-y-publicaciones/noticias-de-socios/265-telefonica-lanza-iniciativa-dereciclaje-la-tierra-te-Ilama-contesta?highlight=WyJkZXNIY2hvcylsImVsZWNOclx1MDBmM25pY29zliwiZGVzZWNob3MgZWxIY3RyXHUwMGYzbmljb3MiXQ

Lauren, J. \& Pennington, J. (2018, 29 de octubre). Tapping the economic value of e-waste. China Daily. http:// europe.chinadaily.com.cn/a/201810/29/WS5bd64e5aa310eff3032850ac.html 
La Prensa Gráfica (2016,4 enero). Incentivan la recolección de desechos electrónicos. La Prensa Gráficahttps://www.laprensagrafica.com/economia/Incentivan-la-recoleccion-de--desechoselectronicos-20160104-0026.html

Forti, V. , Balde, C. P. , Kuehr, R. \& Bel, G. . (2020). The Global E-waste Monitor 2020: Quantities, flows and the circular economy potential. Unión Internacional de Telecomunicaciones, United Nations University \& International Solid Waste Association

http://ewastemonitor.info/wp-content/uploads/2020/07/GEM_2020_def_july1_low.pdf

Foro Económico Mundial. (2019). A New Circular Vision for Electronics: Time for a Global Reboot. Foro Económico Mundial http://www3.weforum.org/docs/WEF_A_New_Circular_Vision_for_Electronics.pdf

Serrano, R. (2019, 19 de diciembre) Nueva Ley De Reciclaje Garantizará Manejo Adecuado De Desechos. Asamblea Legislativa De El Salvador.

https://www.asamblea.gob.sv/node/9840 